\title{
Next Generation of Smart Sensorless Drives for Sustainable Underwater Vehicles
}

\author{
Benedetto Allotta*, Pierluca D'Adamio* \\ Lorenzo Nocentini*, Libero Paolucci*, Luca Pugi*,Andrea Rindi* \\ *Università di Firenze, MDM lab \\ Firenze Italy \\ libero.paolucci@unifi.it
}

\begin{abstract}
Both Autonomous and Remotely Operated Underwater Vehicle play a key role in the development of offshore industrial installations assuring both cost and safety improvements in a very harsh environment. In order to improve performances, reliability and sustainability of underwater robotics, there is the need of a new generation of motors and drives devoted to this kind of applications. In this work authors focus their attention to most interesting aspect of the development of this new generation of products, introducing design examples and results of experimental activities performed on motors installed on Tifone, Marta and Feel Hippo AUVs
\end{abstract}

Keywords-Underwater Robotics; Smart Actuators; Sensorless Control

\section{INTRODUCTION}

Autonomous and Remotely operated under water vehicles, briefly identified with the acronyms "AUV" and "ROV", are extensively used both for under water manipulation and inspection activities. In particular "Remotely operated vehicles" (ROV) have been often used to perform underwater inspection and manipulation activities that have to be periodically performed on off-shore installations and power plants [3], [7]. Also the Autonomous Navigation Capability o AUV is often exploited for long range missions in which the vehicle has to perform long range investigations and data collection [4]. Currently, most of this vehicles are propelled using PM brushless servo-motor whose installation and usage is customized for the underwater environment, resulting in the typical motor configuration visible in Fig. 1 which corresponds to the propulsion layouts adopted as example on Marta [1] or Tifone [2] AUVs, visible in Fig. 2 or on a ROV equipped with manipulators visible in Fig. 3.

Typically in order to reduce the risk of pollution of protected areas the lubricant used to fill the motors should be carefully chosen among a limited set of biodegradable fluids [6]. Also main thermo-mechanical features of the chosen motor has to be identified and re-evaluated considering that the oil filling introduces additional thermal capacities, cooling effects and viscous losses that are completely different from the nominal conditions with respect to the datasheet of the motor. For this reason, electromechanical performances of the motor have to be identified through experimental tests in pool. In order to define these characteristics a test bench is used as shown in Fig. 4.

Starting from the usage of commercial sensorless drive authors focused their research activities in the development of custom sensorless drivers for PM Motors that are highly customized and specialized for under water robotics.

This topic is also relevant from an industrial point of view considering that also major industrial players such as Maxon are starting to develop a dedicated line of products of Oil Filled thrusters for Underwater Applications [8]. In this work, authors focused their attention on the design of a sensorless speed estimator for BLDC motors and on performing experimental activities for the development of this customized application of sensorless drives for underwater robotics.

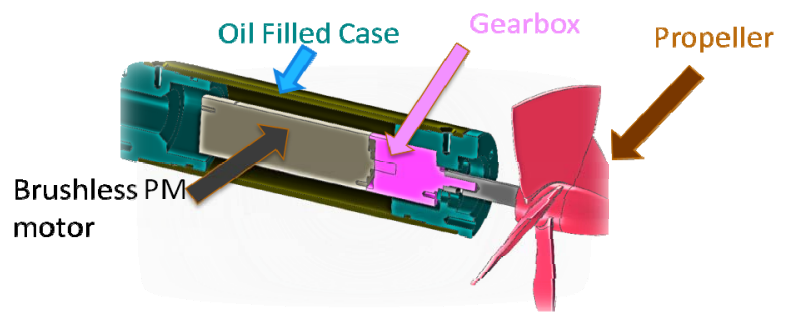

Fig. 1. Oil filled Thrsuter Typical Layout 


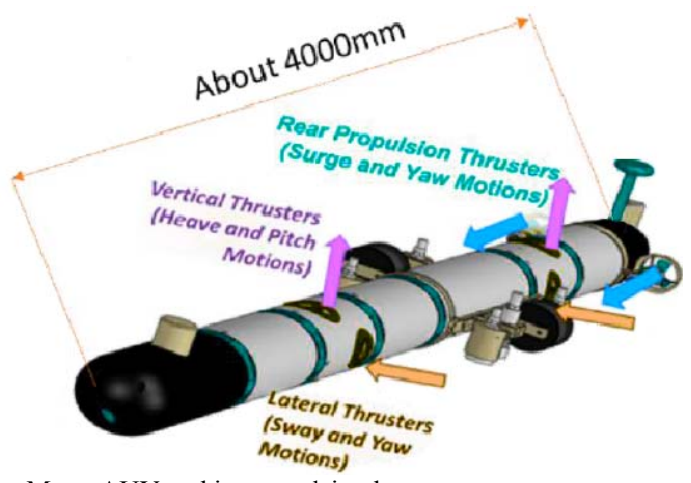

Fig. 2. Marta AUV and its propulsion layout

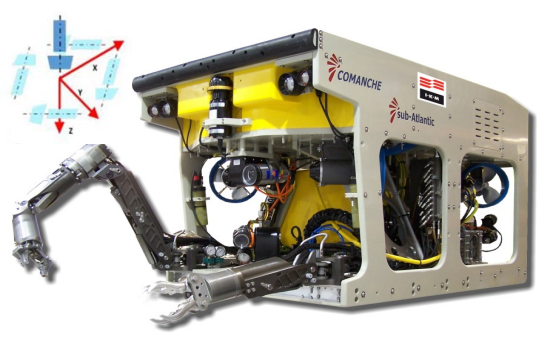

Fig. 3. Work ROV and its typical propulsion layout

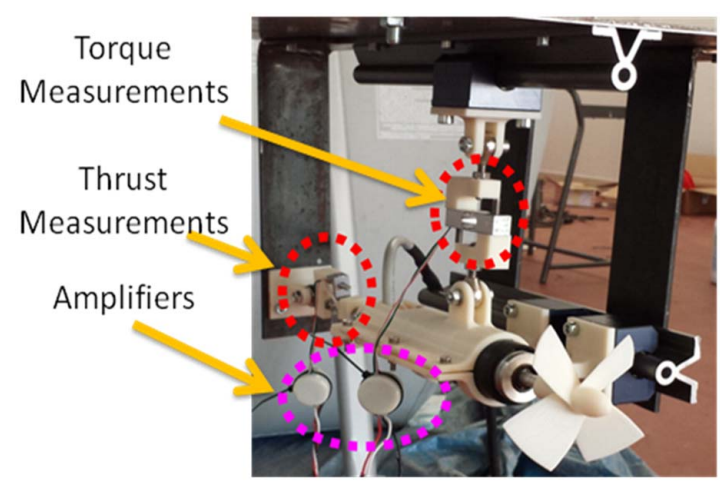

Fig. 4. Experimental test bench in order to indentify of the performances of the assembled Thruster

\section{DRIVER SPECIFICATIONS}

For the development of the sensorless driver authors assumed a feeding DC BUS with a voltage between $12-48 \mathrm{~V}$, and a maximum continuous output current of about 10-20A corresponding to the range of actuators which are more commonly used for under water application of small and medium sized AUVs (from 100 to $800 \mathrm{~W}$ ).

The typical sensorless drive control layout is shown in Fig. 5. The drive is configured with an external speed loop and an internal torque loop.

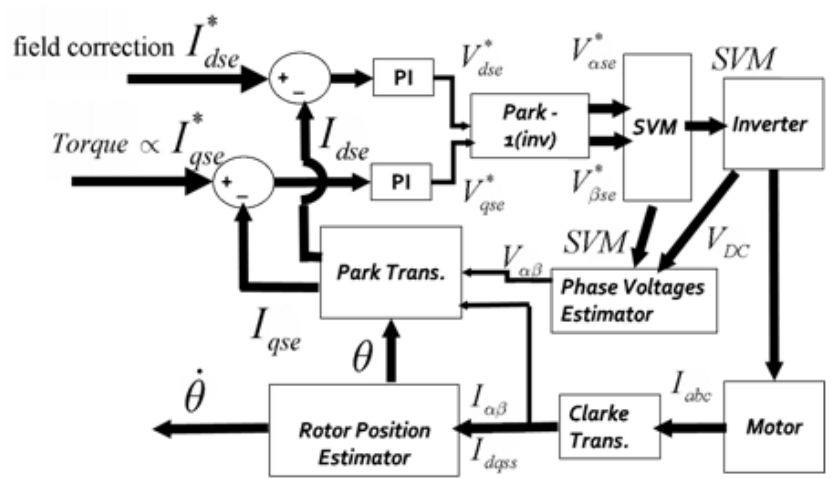

Fig. 5. Typical control layout of sensorless drives

By implementing such control techniques it is also possible to perform speed and torque measurements. For what concerns underwater applications this makes possible the implementation of the following functionalities:

- Vehicle speed estimation: according classical propulsion manuals such as [5] there is a known correlation between propeller speed, propeller torque and relative advance speed in water. In this way, it's possible to implement new functionalities in order to use the motor both to roughly estimate vehicle advance speed and its drag motion resistances.

- Assembled Tuning of the Motor with the possibility of direct thermocouple feedback: the driver is able to perform the tuning of the loop of the assembled actuator, with gearbox, propellers operating in water. In this case it's quite important also the usage of thermocouple integrated in the motor (if available) to evaluate the real thermal limits of the oil filled motor in water.

- Advanced tuning of the sensorless controller: since the motor should be drastically overloaded in terms of currents and temperatures with respect to nominal conditions the sensorless control algorithm has to be tuned in order to be robust against the variability of the main electro-magnetical parameters of the motors such as resistances on windings (due to a variation of temperature) or other non-linearities introduced by high current overloads.

\section{Operation Of SMO BASED SPEEd Estimators}

The typical mathematical model of a PMSM is described, in two-axis $\alpha, \beta$ with stationary reference $(a, b)$, as follows:

$$
\frac{d I_{a}}{d x}=-\frac{R}{L} I_{a}-\frac{1}{L} e_{a}+\frac{1}{L} V_{\alpha}
$$




$$
\begin{gathered}
\frac{d I_{\beta}}{d x}=-\frac{R}{L} I_{\beta}-\frac{1}{L} e_{\beta}+\frac{1}{L} V_{\beta}, \\
e_{\omega}=-\lambda_{0} \omega_{\theta} \sin \theta_{\theta} \\
e_{\beta}=\lambda_{0} \omega_{\theta} \sin \theta_{\theta}
\end{gathered}
$$

where $\mathrm{v}_{\alpha}, \mathrm{v}_{\beta}$ are the $\alpha$ and $\beta$ axis voltages; $\mathrm{i}_{\alpha}, \mathrm{i}_{\beta}$, are the $\alpha$ and $\beta$ axis currents, $\mathrm{R}$ is the phase winding resistance; $\mathrm{L}$ is the inductance; $\omega_{\mathrm{e}}$ is the rotating speed of magnet flux; $\lambda_{0}$ is the permanent magnet flux linkage. According to the motor model it is possible to define an SMO estimator as follows:

$$
\begin{aligned}
& \frac{d \Gamma_{a}}{d x}=-\frac{R}{L} \Gamma_{\alpha}-\frac{1}{L} e_{a}+\frac{L_{1}}{L} \operatorname{sign}\left(\bar{I}_{\alpha}\right) \\
& \frac{d \Gamma_{\beta}}{d x}=-\frac{R}{L} \Gamma_{\beta}-\frac{1}{L} e_{\beta}+\frac{L_{1}}{L} \operatorname{sign}\left(\bar{I}_{\beta}\right)
\end{aligned}
$$

Where $\widehat{\mathbf{I}_{\alpha}}$ and $\widehat{\boldsymbol{F}_{\boldsymbol{\beta}}}$ are estimated currents; $1_{1}$ is a constant observer gain; $\bar{I}_{\boldsymbol{\alpha}}$ and $\bar{I}_{\boldsymbol{\beta}}$ are the observer mismatches $\left(\bar{I}_{\alpha}=\overline{\mathfrak{C}}_{\alpha}-I_{\alpha}, \bar{I}_{\beta}=\bar{f}_{\beta}-I_{\beta}\right)$.

Once the estimator equations are defined it is possible to define a sliding surface as follows:

$$
\begin{array}{ll}
S_{a}=I_{a}-I_{a} & {[\mathrm{~A}]} \\
S_{\beta}=I_{\beta}-I_{\beta} & {[\mathrm{A}]}
\end{array}
$$

According to Sliding Mode theory, error dynamics reaches zero when:

$$
S=0
$$

By selecting the following Lyapunov function:

$$
V=\frac{1}{2} S^{T} S=\frac{1}{2}\left(S_{\alpha}^{2}+S_{\beta}^{2}\right),
$$

It is possible to impose the sliding condition:

$$
S S<0 \text {. }
$$

To do that, it is necessary to define the error dynamics:

$$
\begin{aligned}
& \frac{d S_{\alpha}}{d t}=-\frac{R}{L} S_{\alpha}+\frac{1}{L} e_{\alpha}-\frac{l_{1}}{L} \operatorname{sign}\left(\bar{I}_{\alpha}\right) \\
& \frac{d S_{\beta}}{d t}=-\frac{R}{L} S_{\beta}+\frac{1}{L} e_{\beta}-\frac{L_{1}}{L} \operatorname{sign}\left(\bar{I}_{\beta}\right) .
\end{aligned}
$$

The condition described in (10) is respected by imposing the following:

$$
\iota_{1}>\max \left(\left|e_{\alpha}\right|\left|, e_{\beta}\right|\right)
$$

By verifying the sliding condition the dynamics of the sliding surface and its derivative are:

$$
\left(\begin{array}{c}
S_{a} \\
S_{\beta}
\end{array}\right)=\left(\begin{array}{c}
S_{a} \\
S_{\beta}
\end{array}\right)=0
$$

If

(13) is substituted in

(11) the

following expression of back electromotive force is obtained:

$$
\left\{\begin{array} { l } 
{ 0 = e _ { \alpha } - l _ { 1 } \operatorname { s i g n } ( \overline { I } _ { \alpha } ) } \\
{ 0 = e _ { \beta } - l _ { 1 } \operatorname { s i g n } ( \overline { I } _ { \beta } ) }
\end{array} \rightarrow \left\{\begin{array}{l}
e_{\alpha}=l_{1} \operatorname{sign}\left(\bar{I}_{\alpha}\right) \\
e_{\beta}=l_{1} \operatorname{sign}\left(\bar{I}_{\beta}\right)
\end{array}\right.\right.
$$

Equation (14) can be expressed with the following flow diagram (Fig. 6):

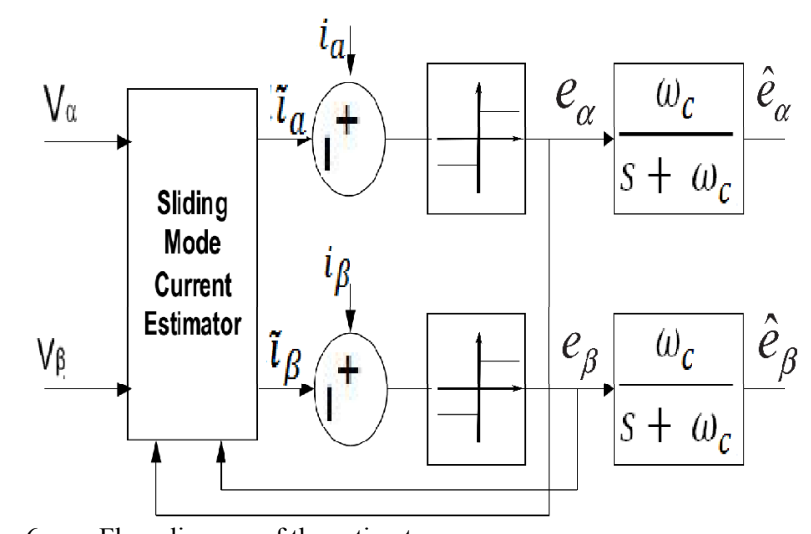

Fig. 6. Flow diagram of the estimator

The technique used to estimate the Back EMF is called bang-bang and, as shown in (14) is implemented by using a $\operatorname{sign}()$ function. So signals $e_{\alpha}$ and $e_{\beta}$ are square waves with a low frequency component consisting in the Back EMF frequency signal and other higher frequency harmonics generated by the switching of the sign() function. Thus, it is necessary to reject these harmonics components in order to make the estimated signal exploitable. In Fig. 6 it is shown the complete diagram of the Back EMF estimator. Signals $e_{\alpha}$ and $e_{\beta}$ are filtered with a low pass filter in order to reject switching harmonics. Clearly the most accurate is the filtering of this signal, the better is the estimation of the Back EMF. For this reason, the cut-off frequency of the low pass filters is a very important parameter in order to optimize SMO based filter operation.

The second part of the estimator, is the Phase Locked Loop. It estimates the rotor speed and position through Back EMF esteem previously generated. The block diagram of the PLL is shown in Fig. 7. From Fig. 7 it we have: 


$$
\begin{gathered}
\left.\Delta e=\hat{\varepsilon}_{\alpha} \cos \hat{\theta}-\hat{\varepsilon}_{\beta} \sin \hat{\theta}\right) \\
=\lambda_{0} \omega_{\Theta} \sin \hat{\theta}_{\theta} \cos \hat{\theta}-\lambda_{0} \omega_{\Theta} \sin \hat{\theta}_{\Theta} \sin \hat{\theta}
\end{gathered}
$$$$
=\lambda_{0} \omega_{\theta} \sin \left(\hat{\theta}_{\theta}-\hat{\theta}\right) \text {. }
$$

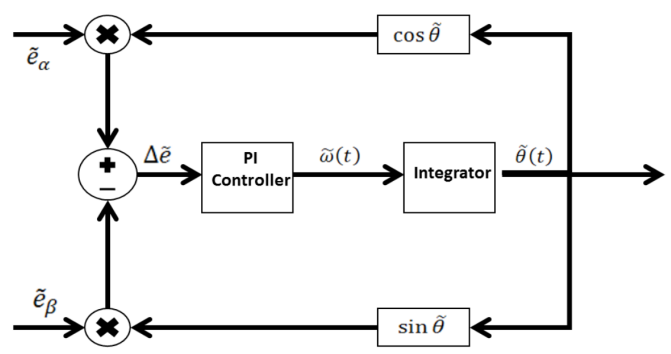

Fig. 7. Flow diagram of the PLL

\section{EXPERIMENTAL ACTIVITIES, DEVELOPMENT TOOLS AND RESULTS}

One of the sub-system to which authors have dedicated more attention referring to the scheme of Fig. 5 is represented by the Sensorless Rotor Estimator. For the proposed application, the control of a marine propeller it's not important to accurately control the motor a speed lower than $10 \%$ of the nominal one. For these reason authors started their work considering a classical Back-EMF estimator which have been customized and enhanced in terms of robustness and performances for the proposed application which corresponds to the scheme of Fig. 5 and Fig. 6. In particular authors focused their attention on the combined application of Sliding Mode Observer [10], Phase Locked Loop filters [9] testing also the possibility of a gain scheduling with respect to the observed states.

In particular the activity has been performed according to the following steps:

- Preliminary prototyping and off-line simulation with Simulink-Simscape ${ }^{\mathrm{TM}}$ libraries

- Preliminary fast prototyping on a DSPACE AUTOBOX 1513 RT Controller

- Preliminary implementation and testing using FPGA module of NI Compact RIO

- Final Implementation and testing both on TI (C2000) and on a Microchip PIC32MZ2048ECH100 micro-controller.

For what concerns the preliminary Simulink-Simscape ${ }^{\mathrm{TM}}$ based simulations, voltage and current waveforms were generated by a mathematical model of a BLDC motor.

The other tests have been performed on a real motor by acquiring voltage and current measurements while it was spinning. The motor was controlled by a Texas Instruments drive system, the Piccolo LAUNCHXL-F28027F controller + BOOSTXL-DRV8301.

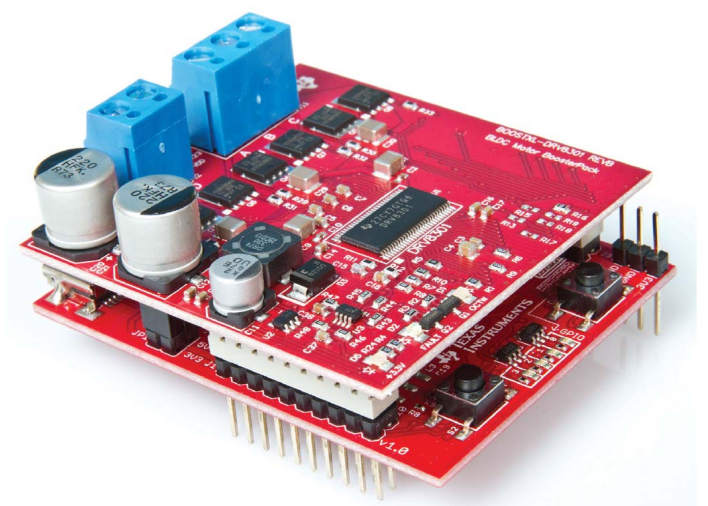

Fig. 8. Piccolo LAUNCHXL-F28027F controller + BOOSTXL-DRV8301

The final implementation of the SMO based filter algorithm has been implemented in an embedded system in $\mathrm{C}$ language. The use of low cost and small size microcontrollers such as the Microchip PIC32MZ2048ECH100 is meant to be a solution in order to achieve an optimal integration of motor and control logic. On this purpose, a test board equipped with the PIC32MZ2048ECH100 microcontroller has been developed.

Thanks to this system, it has been possible to acquire and process the voltage and current signals on the three phases of the motor in order to test the SMO estimator.

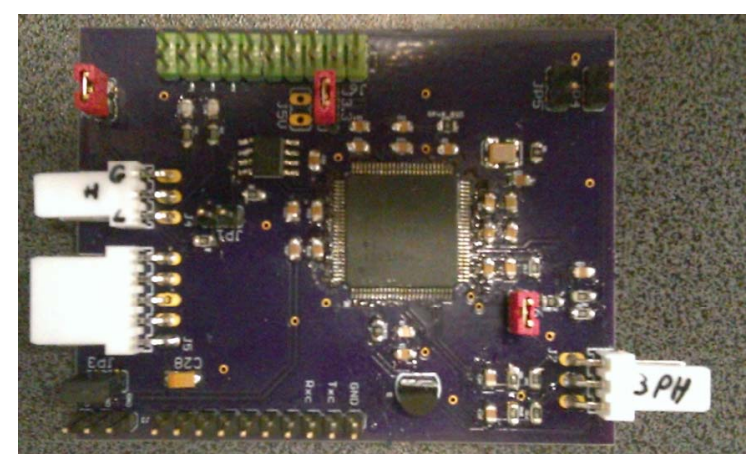

Fig. 9. SMO filter test board equipped with PIC32MZ2048ECH100

All the activities have been performed using the same actuator [8] used for the propulsion of Marta AUV. The LAUNCHXLF28027F board is programmed with a sensorless control technique for BLDC motors, which is also based on a SMO filter. We will refer to this filter as TI filter and the developped solution as the SMO filter. Although the complete control algorithm is not accessible for commercial reasons, the TI filter could be considered a good reference in order to compare the performances of the developed solution. On this purpose authors have developed a Matlab based acquisition interface. Thanks to this interface, it is possible to acquire in real-time the estimated values (rotor position, speed, and torque) from both the TI filter and the author's developed solution, to vary filter parameters and to execute comparative tests.

Authors have characterized and studied the filter in terms of robustness and overall performance over a wide range of 
speed (from standstill until $20000 \mathrm{rpm}$ ). Since authors develop solutions for underwater applications, tests have been also been performed in water in order to determine the performances of the system in harsh conditions.

Some experimental results of the comparison of the two filters are visible in Fig. 10, Fig. 11, Fig. 12 and Fig. 13. It's important to underline the fact that TI filter estimated speed is feed back to the controller in order to perform motor control. For this reason, reference speed and estimated speed coincide. For what concerns the SMO filter, it operates in open loop, so the two measurements may differ.
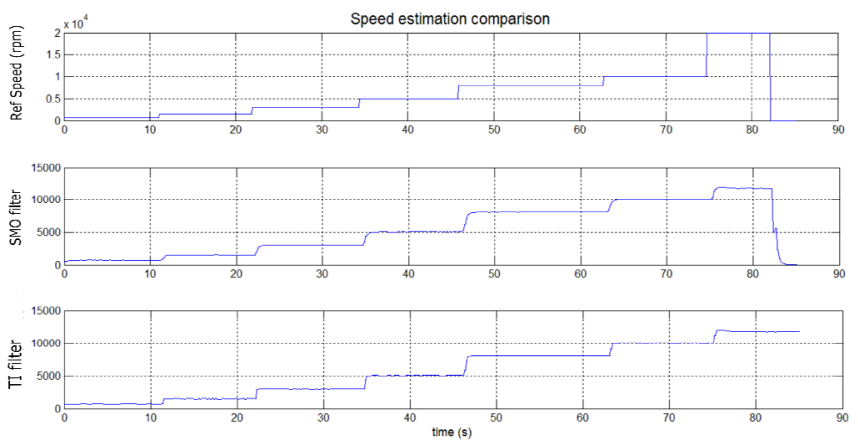

Fig. 10. Estimated speed according to a stepped speed reference profile

In Fig. 10 it is shown a comparison between the TI filter estimated speed and the SMO filter, according to a stepped speed reference profile. The overall performance of the two filter is similar. By zooming in the graph, it is possible to notice that the ripple amplitude, which affects the measured signal, varies according to the measured speed for both the TI and the SMO filter. In the performed test, reference speed has been span from 700 to $12000 \mathrm{rpm}$. In Fig. 10 the SMO filters uses a cut-off frequency filter for the PLL of 10rad/s. PLL filter is shown in Fig. 6.

When dealing with sensorless control systems the most critical task, is controlling it at low speed with respect to the nominal speed. This because in general, sensorless techniques, to estimate speed and position of the rotor, exploit back electromotive force induced in stator coils during rotation, which decreases at low speed. The motor used for the tests, has a nominal speed of $25000 \mathrm{rpm}$ so speed estimation is critical when reference speed is below $2000 \mathrm{rpm}$.

In Fig. 11 it is shown the measurement error of the two filters given a reference speed of $700 \mathrm{rpm}$. In this case the SMO filter seems to perform better than the TI filter, although from Fig. 10 it's clearly visible how the SMO filter is slower when chasing a speed variation.

The behavior of the two filters is also highlighted in TABLE 1. In there it has been computed the standard deviation of the speed measurement error at the various speed step. The two filters have similar performances at high speed while from 700 to $3000 \mathrm{rpm}$ the SMO filter performs better in terms of average speed error.

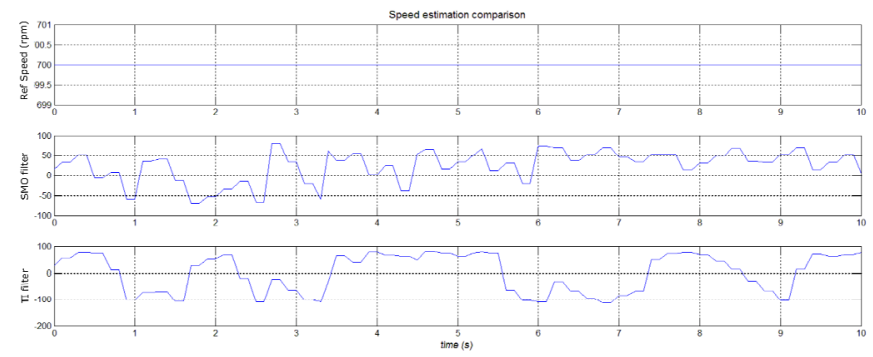

Fig. 11. Speed measurement error of the two filters given a reference speed of $700 \mathrm{rpm}$

At higher speed ripple amplitude decrease regardless of the PLL filter cut-off frequency as shown in Fig. 12 and Fig. 13. Using a low-pass filter frequency of $50 \mathrm{rad} / \mathrm{s}$ to estimate speed lower than $2000 \mathrm{rpm}$, results in a very noisy speed estimation.

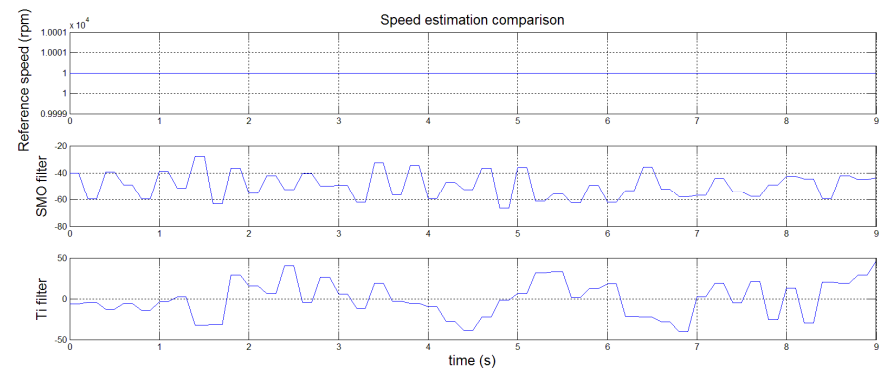

Fig. 12. Speed measurement error of the two filters given a reference speed of $10000 \mathrm{rpm}$ and $10 \mathrm{rad} / \mathrm{s}$ PLL filter

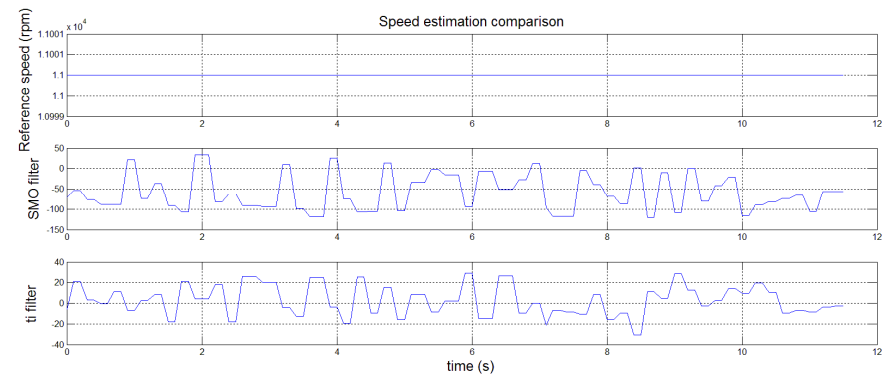

Fig. 13. Speed measurement error of the two filters given a reference speed of $11000 \mathrm{rpm}$ and 50rad/s PLL filter

TABLE 1.

\begin{tabular}{|c|c|c|}
\hline & \multicolumn{2}{|c|}{$\begin{array}{c}\text { Standard deviation of the speed } \\
\text { measurement error according to } \\
\text { different measured speed }\end{array}$} \\
\hline & $\begin{array}{c}\text { SMO filter } \\
\text { (10rad/s LPF) }\end{array}$ & TI filter \\
\hline $\mathbf{7 0 0} \mathbf{r p m}$ & 39.0818 & 71.7796 \\
\hline $\mathbf{1 5 0 0} \mathbf{~ r p m}$ & 24.6550 & 77.3445 \\
\hline $\mathbf{3 0 0 0} \mathbf{~ r p m}$ & 16.3979 & 35.1901 \\
\hline $\mathbf{5 0 0 0} \mathbf{~ r p m}$ & 15.9772 & 10.2908 \\
\hline $\mathbf{8 0 0 0} \mathbf{~ r p m}$ & 13.3922 & 10.9181 \\
\hline $\mathbf{1 0 0 0 0} \mathbf{~ r p m}$ & 10.9927 & 20.2238 \\
\hline
\end{tabular}

In order to keep the system ready and optimize the performances of the filter, the proposed solution in thus a 
scheduling of the PLL cut-off frequency filter according to the reference speed.

\section{CONCLUSIONS}

The developed sensorless speed and position estimator, has interesting performances over a wide range of speed. It's also interesting for what concerns hardware costs which are extremely low. The application of modern code prototyping techniques to smart and cheap micro-controllers can made possible the design of a customized sensorless drive system that will substitute less performing and more expensive commercial solutions previously adopted on Marta and Feel Hyppo AUVs.

Having the possibility of monitoring in real-time motor operation, rotor speed and delivered torque opens up new perspectives in order to improve underwater vehicles navigation. The described test have been performed being the motor and the propeller docked to a test bench. Further test will be carried on by recording measurements of the motor operating in a navigating vehicle. Torque, and speed information gathered in real-time form the motor driver, could be exploited in order to optimize and determine vehicle autonomy and help advance speed estimation.

\section{ACKNOWLEDGEMENTS}

This activity was partially supported and financed by the European project ARROWS ( $7^{\text {th }}$ European Framework Program http://www.arrowsproject.eu/) which financed the realization of the Marta AUV including its propulsion system. Authors wish also to thank for their precious support all the people of TI University Program and in particular Nuria Llin, the manager of the TI university program which have supported author efforts with precious resources both in terms of components (microcontrollers and semiconductors) and technical know-how (support during the final implementation phase).

\section{REFERENCES}

[1] Allotta, B., Costanzi, R., Gelli, J., Pugi, L., \& Ridolfi, A. (2015, May). Design of a modular propulsion system for MARTA AUV. In OCEANS 2015-Genova (pp. 1-7). IEEE.

[2] Allotta, B., Pugi, L., Bartolini, F., Ridolfi, A., Costanzi, R., Monni, N., Gelli, J. Preliminary design and fast prototyping of an Autonomous Underwater Vehicle propulsion system (2015) Proceedings of the Institution of Mechanical Engineers Part M: Journal of Engineering for the Maritime Environment, 229 (3), pp. 248-27 DOI: $10.1177 / 1475090213514040$

[3] Romanowicz, B., Stakes, D., Uhrhammer, R., McGill, P., Neuhauser, D., Ramirez, T., \& Dolenc, D. (2003). The MOBB experiment: A prototype permanent off-shore ocean bottom broadband station. Eos, Transactions American Geophysical Union, 84(34), 325-332.J. Clerk Maxwell, A Treatise on Electricity and Magnetism, 3rd ed., vol. 2. Oxford: Clarendon, 1892, pp.68-73.

[4] Caress, D. W., Thomas, H., Kirkwood, W. J., McEwen, R., Henthorn, R., Clague, D. A., ... \& Maier, K. L. (2008). High-resolution multibeam, sidescan, and subbottom surveys using the MBARI AUV D. Allan B. Marine habitat mapping technology for Alaska, 47-69.

[5] Carlton, J. (2012). Marine propellers and propulsion. ButterworthHeinemann.

[6] Nagendramma, P., \& Kaul, S. (2012). Development of ecofriendly/biodegradable lubricants: An overview. Renewable and Sustainable Energy Reviews, 16(1), 764-774.

[7] Parker, R. M., Harrison, G. P., \& Chick, J. P. (2007). Energy and carbon audit of an offshore wave energy converter. Proceedings of the Institution of Mechanical Engineers, Part A: Journal of Power and Energy, 221(8), 1119-1130.

[8] http://www.maxonmotor.it/ , Hall Heavy Duty - Oil Filled Application Code 426451

[9] Hsieh, G. C., \& Hung, J. C. (1996). Phase-locked loop techniques. A survey.Industrial Electronics, IEEE Transactions on, 43(6), 609-615.

[10] Kim, H., Son, J., \& Lee, J. (2011). A high-speed sliding-mode observer for the sensorless speed control of a PMSM. Industrial Electronics, IEEE Transactions on, 58(9), 4069-4077. 